\title{
The Dynamic of Staphylococcus aureus Nasal Carriage in Central Iran
}

\author{
Nasimeh Fard-Mousavi ${ }^{1}$; Ghasem Mosayebi ${ }^{1}$; Alireza Amouzandeh-Nobaveh ${ }^{1}$; Alireza \\ Japouni-Nejad ${ }^{1}$; Ehsanollah Ghaznavi-Rad ${ }^{2, *}$
}

${ }^{1}$ Department of Medical Microbiology and Immunology, Faculty of Medicine, Arak University of Medical Sciences, Arak, IR Iran

2 Molecular Research Center, Faculty of Medicine, Arak University of Medical Sciences, Arak, IR Iran

${ }^{*}$ Corresponding author: Ehsanollah Ghaznavi-Rad, Molecular Research Center, Faculty of Medicine, Arak University of Medical Sciences, Arak, IR Iran. Tel: +98-8634173526, Fax: +98-8634173526, E-mail: e.ghaznavirad@arakmu.ac.ir

Received: May 28, 2014; Revised: August 16, 2014; Accepted: August 28, 2014

\begin{abstract}
Background: Nasal carriage of Staphylococcus aureus plays an important role in the pathogenesis of staphylococcal infections. Anterior nasal region is a primary origin of S. aureus. In longitudinal studies, three types of S. aureus nasal carriers can be distinguished: persistent carriers, intermittent or transient carriers, and noncarriers.

Objectives: This study was designed to determine the dynamic of S. aureus nasal carriage in healthy carriers of central Iran.

Patients and Methods: A total of 813 healthy adults were subjected to this cross-sectional study from November 2011 to January 2012 in Arak University of Medical Sciences. Two anterior nasal swabs were taken with a week interval from each participant. All the isolates were identified as S. aureus phenotypically by standard laboratory methods. The isolates were reconfirmed by amplification of sa442 gene as the identification marker. All the isolates were screened for the presence of the PVL (Panton-Valentine leukocidin) virulence genes and arginine catabolic mobile element (ACME-arc).

Results: Among the 813 subjects screened, 83 (10.2\%) were persistent carriers, 86 (10.6\%) were transient carriers and 644 (79.2\%) cases were found as noncarriers. A total of 169 (20.8\%) participants had colonized S. aureus. The frequency of ACME-arc A and PVL genes in S. aureus strains were $17 \%$ and $20 \%$, respectively.

Conclusions: Carriage of PVL-positive S. aureus is common in this region, even in the low frequency of MRSA colonization. The detection of ACME-arcA gene in S. aureus isolates is a public-health concern and demands continued surveillance and close monitoring.

Keywords: Staphylococcus aureus; Panton-Valentine Leukocidin; Dynamics Population
\end{abstract}

\section{Background}

Staphylococcus aureus is a common commensal of humans as well as an important cause of infectious diseases such as skin and soft tissue infections, blood stream infections, pneumonia, osteomyelitis, and endocarditis $(1,2)$. Staphylococcus aureus colonizes in various parts of healthy humans' bodies such as the nares, skin and vagina, but studies have shown that the anterior nasal region is the most consistent area in which this organism can be isolated $(2,3)$. Nasal colonization is an important stage in the pathogenesis of $S$. aureus infection and is a risk factor for infections in both hospitals and the community (4). Longitudinal studies have distinguished three S. aureus nasal carriage patterns in healthy individuals, which includes persistent carriers, intermittent carriers, and noncarriers (3). Persistent carrier is a person whose nose culture is positive within a one-week interval or contains $10^{3}$ colony forming units; if only one of the cultures is positive or the colony-forming unit (CFU) is less than 103, the person will be considered an intermediate carrier. A noncarrier is a person whose nasal cultures are all negative for $S$. aureus $(5,6)$.
The prevalence of $S$. aureus nasal carriage varies and is higher in young children (7), hospitalized patients, males (8), and a number of patients including patients with diabetes mellitus (9), chronic ambulatory diseases, those with $S$. aureus skin infection (10), and HIV-infected patients $(6,11)$. There is evidence that certain individuals may carry their resident strain for long periods, sometimes even for years (11). Besides, von Eiff and colleagues have shown that $80 \%$ of $S$. aureus infections in bacteria carriers are attributable to an endogenous source (12). The statistics used to identify these carriage patterns have been opposed. Approximately, 10\% to 35\% of healthy individuals are almost always persistent carriers and intermittent carriers are between $20 \%$ and $75 \%$; eventually, $5-50 \%$ of healthy individuals become noncarriers (3, 6 , 13-15). The ability of $S$. aureus strains to produce a diverse range of diseases depends on the expression of several virulence factors.

Panton-Valentine leukocidin (PVL) is considered as one of the most important virulence determinants of $S$. aureus and community-acquired methicillin-resistant $S$.

Copyright (C) 2015, Ahvaz Jundishapur University of Medical Sciences. This is an open-access article distributed under the terms of the Creative Commons Attribution-NonCommercial 4.0 International License (http://creativecommons.org/licenses/by-nc/4.0/) which permits copy and redistribute the material just in noncommercial usages, provided the original work is properly cited. 
aureus (CA-MRSA). PVL is a bicomponent toxin that forms polymeric pores in leukocyte membranes and plays an important role in the pathogenesis of necrotizing pneumonia and clinical syndromes of necrotic skin lesions (furuncles) $(5,16,17)$. Arginine catabolic mobile element (ACME) is a proposed virulence gene and the survival factor of S. aureus, which was discovered by whole genome sequencing of USA300 (ST-8). Acquisition of ACME by S. aureus, a transient colonizer of the skin, may allow CAMRSA to colonize the skin on a permanent basis, thereby enhancing the likelihood of skin infection $(18,19)$. There is no study to determine the pattern of S. aureus nasal carriage in Iranian healthy carriers.

\section{Objectives}

The aim of this study was to determine the dynamic of S. aureus nasal carriage and explore the frequency of $P V L$ and ACME virulence genes among healthy carriers in central Iran.

\section{Patients and Methods}

\subsection{Isolation and Characterization of Staphylococ- cus aureus}

A cross-sectional study was performed on 813 volunteers from November 2011 to May 2012 and was approved by the Ethics Committee of Arak University of Medical Sciences campus after institutional ethical approval (No. 3-143-1392). Students without symptoms or signs of clinical illnesses and antibiotic consumption were enrolled in the study.

Sampling was performed twice within a week on volunteers. The anterior nasal swabs were collected using Transwab (Medical Wire and Equipment Company, Corsham, UK) and were immediately transported to the laboratory. The nasal swabs were enriched with $7.5 \%$ salt nutrient broth (Merck, Germany) at $37^{\circ} \mathrm{C}$ for $2-4$ hours, then were plated on a Columbia blood agar (Merck, Germany) medium plate and incubated at $37^{\circ} \mathrm{C}$ for 24 hours. Identification of $S$. aureus was based on colony morphology and results of Gram staining. The isolated colony was inoculated on sterilized phenol-red mannitol salt agar (MSA) (Merck, Germany) plates and incubated at $37^{\circ} \mathrm{C}$ for 24 hours. Isolates positive for catalase test, MSA plate, slide coagulase and tube coagulase with human plasma and DNase test and thermostable nuclease, were considered as S. aureus in this study. In addition, all the isolates were checked for the presence of the sa442 gene by PCR (4) (Table 1).

3.2. Detection of Arginine Catabolic Mobile Element (ACME) and Panton-Valentine Leukocidin (PVL) Virulence Genes by Polymerase Chain Reaction

DNA extraction kit (BioFlux, Bioer, Korea) was used for the extraction of genomic DNA from all the S. aureus strains, according to the manufacturer's instructions. PCR was performed for amplification of genes encoding PVL and ACME-arcA (20) (Table 1). Each of the PCR products were sent for sequencing (Gene Fanavaran, Iran) and confirmed through the basic local alignment search tool (BLAST) search before the final results were analyzed.

\section{Results}

Of 813 participants, 83 (10.2\%) and 86 (10.6\%) were persistent and transient carriers, respectively; the population of the overall nasal carriers of S. aureus was $20.8 \%$ (169/813). None of the carriers were ever hospitalized or ever experienced fever during the last two weeks. These comprised 61 males (36\%) and 108 females (64\%), 18 to 44 years old (mean age: $23.5 \pm 5.5$ ). Out of 813 candidates, $644(79.2 \%)$ were noncarriers. All the isolates showed positive results for the amplification of Sa442 identification marker (Figure 1).

Table 1. Profile Primers and Polymerase Chain Reaction Conditions ${ }^{a}$

\begin{tabular}{lcc}
\hline Gene, Primer/Sequence & PCR Product Size & Reference \\
\hline Sa442 & $108 \mathrm{bp}$ & $(4)$ \\
F: AATCTTTGTCGGTACACGATATTCTTCACG & & $(4)$ \\
R: CGTAATGAGATTTCAGTAGATAATACAACA & $433 \mathrm{bp}$ & $(21)$ \\
\hline PVL & & \\
F: ATCATTAGGTAAAATGTCTGGACATGATCCA & $770 \mathrm{bp}$ \\
R: GCATCAATGTATTGGATAGCAAAAGC & \\
\hline ACME & & \\
F: CACGTAACTTGCTAGAACGAG & & \\
R: GAGCCAGAAGTACGCGAG & & \\
\hline
\end{tabular}

\footnotetext{
a The PCR condition was 30 seconds in $95^{\circ} \mathrm{C}, 30$ seconds in $55^{\circ} \mathrm{C}$ and 45 seconds in $72^{\circ} \mathrm{C}$.
} 
Surprisingly, a high frequency of PVL gene among the S. aureus isolates was found, which was 34 (20\%) (Figure 2). In addition, 29 (17\%) were positive for ACME-arc A gene (Figure 3).

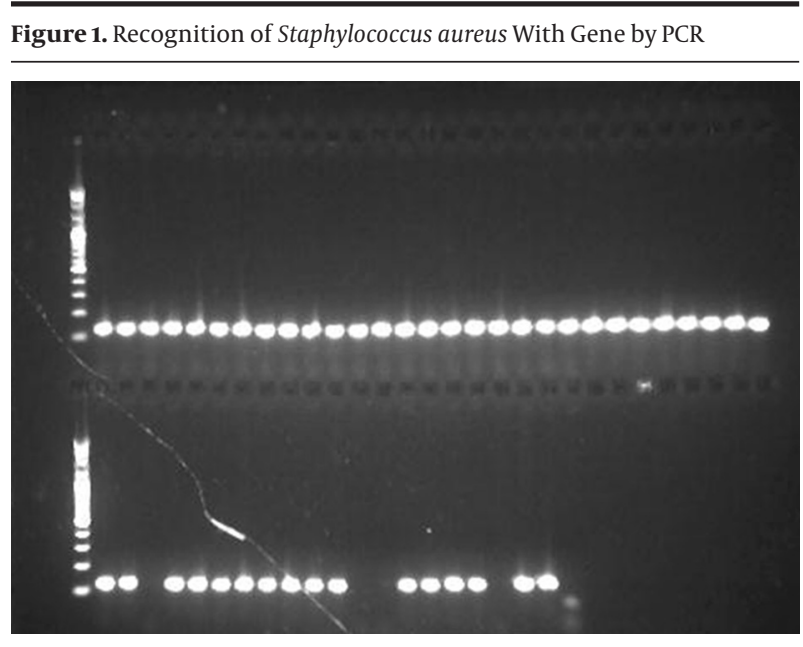

The left lane is weight marker.

Figure 2. Electrophoresis of the Polymerase Chain Reaction Product of the PVL Gene

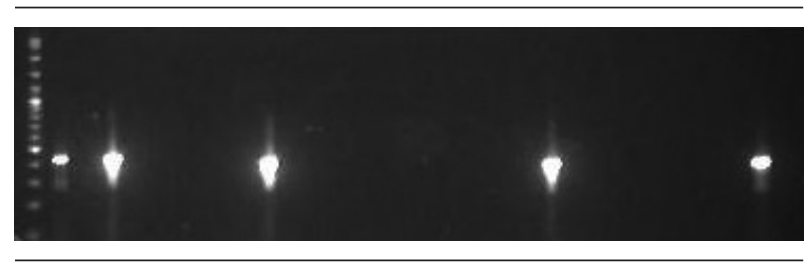

Product size of the PVL gene was $433 \mathrm{bp}$.

Figure 3. Electrophoresis of the Polymerase Chain Reaction Product of the ACME Gene

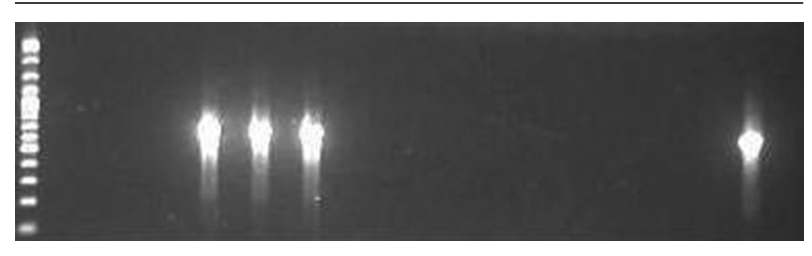

The product size was $770 \mathrm{pb}$.

\section{Discussion}

This study was designed, because most of the studies have been conducted in Iran concerning the carriage of $S$. aureus in healthcare workers and no information exists about the dynamics and even the frequency of $S$. aureus carriage in the healthy population. Therefore, a decision was made to study the dynamics of $S$. aureus carriers in center of Iran and to evaluate the potential risks such as the presence of $P V L$ and ACME genes. The results indicated that generally (20.8\%), healthy people who are in this region are frequent carriers of $S$. aureus. The rate of nasal carriage of S. aureus was $31.1 \%$ in Brazil (21) and in a study performed in Hamedan (Iran), this rate was $25.2 \%$ (22). In another study in Iran, the frequency of nasal carriage at the admission time in pediatrics hospital was $20.7 \%$ (23).

In a study by Seng Choi et al. in Malaysia, the carriers' rate was $23.4 \%(24)$. In general, the colonization rate of $S$. aureus in healthy individuals has been reported $10-70 \%$ in most parts of the world (6). Nevertheless, factors such as age, gender, alcoholism, smoking and taking pregnancy medicines as well as some chronic diseases such as diabetes and skin diseases are considered to increase S. aureus nasal carriage $(9,25)$. In this study, the rate of $S$. aureus carriers among healthy individuals was $20.8 \%$, which was in agreement with the above studies. However, this rate cannot be generalized, as the recent study was performed in a small scale among university students and the number of persons who carry nasal S. aureus may depend on the population under the study. Surprisingly, 83 (49\%) of our healthy carriers were persistence carriers. The explanation could be that persistent carriers are the high-risk group for $S$. aureus infection. This finding in our region must be taken seriously; however, longitudinal follow-up would enable the assessment of the infection frequency in this group.

Although knowledge regarding the host and bacterial factors affecting Staphylococcus colonization is increasing, the reason why some individuals are persistent carriers and others are intermittent carriers or noncarriers is still unclear. However, persistent and intermittent carriers are at a higher risk of exposure to $S$. aureus infections compared to noncarriers $(12,26)$. In addition, differences in procedures of nasal swabbing and isolation of S. aureus may account for some variations in carriage rates. For instance, transmission medium and the incubation period are considerable; it has been documented that swabs from the anterior nares (i.e. the vestibulum nasi) yield higher carriage rates than swabs taken from sites beyond this region (27). In the current study, the main pattern of $S$. aureus carriers belonged to noncarriers, the rate of which is compatible to studies performed by Vasantha Kumari in Malaysia (28). In the study of Eriksen et al. the rate of carriers was high (84\%) and the rate of $S$. aureus carriers in males was higher than females; this proportion was in contrast with our observations (13).

The frequencies of two important virulence genes, PVL and $A C M E$, were determined in the recent study. In our study, PVL was found in $20.8 \%$ of isolates, while in different studies in Turkey, the PVL gene was detected in $0-12.7 \%$ of the isolates (29-33). Around 10\% of S. aureus isolates reported in both the USA and Indonesia have been PVL positive $(34,35)$. Higher prevalence of the PVL gene was found $19.4 \%$ in Malaysia , 19.7\% in Iran and 31\% in New Zealand (36-38). In this study, it was $20 \%$. Generally, PVL-positive S. aureus exists in children and young adults (35). Generally, $P V L$ gene represents a stable marker for CA-MRSA and very 
seldom in HA-MRSA. In Iran, the frequency of PVL gene in HA-MRSA has been found as $4.57 \%$, which is high compared with other parts of the word $(39,40)$. However, only 1 (14.3) out of seven CA-MRSA isolates from healthy carriers harbors the PVL leukocidin gene (20). Increase in PVL rate is becoming critical and requires more attention. The transmission of S. aureus in universities and other crowded places where people are more often in close physical contact with each other can make an endogenous supply for infections, or can help in transmitting and spreading the strain among people. A future research is needed to obtain information about the gene spread of this dangerous isolate among nasal carriers.

Regarding the ACME virulence gene, the study of Francois Barbier et al. found that in Istanbul, the rate of $A C M E$ was $65.4 \%$ (41). In another study by Shore et al. 23 of 238 $A C M E$ isolated $S$. aureus were positive (42). In studies by Ellington et al. and Montgomery et al. the rates of $A C M E$ was $34.5 \%$ and $75.44 \%$, respectively $(43,44)$. In this study, the frequency of the ACME-arc gene was $17 \%$, which was the less than other studies. Although it is believed that $A C M E$ enhances the pathogenicity of the $S$. aureus strains through unknown mechanism, some studies have mentioned that the presence of this gene metabolically alters the local pH on the skin, hence, increases the ability of this microorganism to persist on intact skin (18).

In conclusion, the high prevalence of persistent $S$. aureus carriers and the considerable frequency of the $P V L$ leukocidin gene in our region are worrisome and must be considered as major concerns. Further study is needed to investigate the relationship between S. aureus nasal colonization and infection, through molecular epidemiology of S. aureus isolated from carriers and infection sites.

\section{Acknowledgements}

This article was a part of the thesis (No. 861) by Nasimeh Fard-Mousavi to fulfill the requirement for a Master of Sciences in microbiology. The Authors acknowledge the Vice Chancellor for Research at Arak University of Medical Sciences for financial support of the research.

\section{Authors' Contributions}

Study concept and design: Ehsanollah Ghaznavi-Rad, Ghasem Mosayebi, Nasimeh Fard-Mousavi. Laboratory work, analysis and interpretation of data: Nasimeh FardMousavi, Alireza Japouni-Nejad. Drafting of the manuscript: Nasimeh Fard-Mousavi and Alireza Amouzandeh. Critical revision of the manuscript: Ehsanollah GhaznaviRad and Ghasem Mosayebi.

\section{Financial Disclosure}

All the authors declared that they had no financial interests related to the materials in the manuscript.

\section{Funding/Support}

This study was conducted with financial assistance from Arak University of Medical Sciences, Iran.

\section{References}

1. Richards MJ, Edwards JR, Culver DH, Gaynes RP. Nosocomial infections in medical intensive care units in the United States. National Nosocomial Infections Surveillance System. Crit Care Med. 1999;27(5):887-92.

2. Perez-Vazquez M, Vindel A, Marcos C, Oteo J, Cuevas O, Trincado P, et al. Spread of invasive Spanish Staphylococcus aureus spa-type t067 associated with a high prevalence of the aminoglycosidemodifying enzyme gene ant( $\left.4^{\prime}\right)$-Ia and the efflux pump genes msrA/msrB. J Antimicrob Chemother. 2009;63(1):21-31.

3. Williams RE. Healthy carriage of Staphylococcus aureus: its prevalence and importance. Bacteriol Rev. 1963;27:56-71.

4. Martineau F, Picard FJ, Roy PH, Ouellette M, Bergeron MG. Speciesspecific and ubiquitous-DNA-based assays for rapid identification of Staphylococcus aureus. J Clin Microbiol. 1998;36(3):618-23.

5. Lina G, Piemont Y, Godail-Gamot F, Bes M, Peter MO, Gauduchon $\mathrm{V}$, et al. Involvement of Panton-Valentine leukocidin-producing Staphylococcus aureus in primary skin infections and pneumonia. Clin Infect Dis. 1999;29(5):1128-32.

6. Wertheim HFL, Melles DC, Vos MC, van Leeuwen W, van Belkum A Verbrugh HA, et al. The role of nasal carriage in Staphylococcus aureus infections. Lancet Infect Dis. 2005;5(12):751-62.

7. Peacock SJ, Justice A, Griffiths D, de Silva GD, Kantzanou MN Crook D, et al. Determinants of acquisition and carriage of Staphylococcus aureus in infancy. J Clin Microbiol. 2003;41(12):5718-25.

8. Cole AM, Tahk S, Oren A, Yoshioka D, Kim YH, Park A, et al. Determinants of Staphylococcus aureus nasal carriage. Clin Diagn Lab Immunol. 2001;8(6):1064-9.

9. Lipsky BA, Pecoraro RE, Chen MS, Koepsell TD. Factors affecting staphylococcal colonization among NIDDM outpatients. Diabetes Care. 1987;10(4):483-6.

10. Hoeger PH, Lenz W, Boutonnier A, Fournier JM. Staphylococcal skin colonization in children with atopic dermatitis: prevalence, persistence, and transmission of toxigenic and nontoxigenic strains. J Infect Dis. 1992;165(6):1064-8.

11. Ho PL, Wang TK, Ching P, Mak GC, Lai E, Yam WC, et al. Epidemi ology and genetic diversity of methicillin-resistant Staphylococcus aureus strains in residential care homes for elderly persons in Hong Kong. Infect Control Hosp Epidemiol. 2007;28(6):671-8.

12. von Eiff C, Becker K, Machka K, Stammer H, Peters G. Nasal carriage as a source of Staphylococcus aureus bacteremia. Study Group. N Engl J Med. 2001;344(1):11-6.

13. Eriksen NH, Espersen F, Rosdahl VT, Jensen K. Carriage of Staphylococcus aureus among 104 healthy persons during a 19-month period. Epidemiol Infect. 1995;115(1):51-60.

14. Hu L, Umeda A, Kondo S, Amako K. Typing of Staphylococcus aureus colonising human nasal carriers by pulsed-field gel electrophoresis. J Med Microbiol. 1995;42(2):127-32.

15. Nouwen JL, Fieren MW, Snijders S, Verbrugh HA, van Belkum A Persistent (not intermittent) nasal carriage of Staphylococcus aureus is the determinant of CPD-related infections. Kidney Int 2005;67(3):1084-92.

16. Gillet Y, Issartel B, Vanhems P, Fournet JC, Lina G, Bes M, et al Association between Staphylococcus aureus strains carrying gene for Panton-Valentine leukocidin and highly lethal necrotising pneumonia in young immunocompetent patients. Lancet. 2002;359(9308):753-9.

17. Kaneko J, Kamio Y. Bacterial two-component and hetero-heptameric pore-forming cytolytic toxins: structures, pore-forming mechanism, and organization of the genes. Biosci Biotechnol Biochem. 2004;68(5):981-1003.

18. Diep BA, Gill SR, Chang RF, Phan TH, Chen JH, Davidson MG, et al. Complete genome sequence of USA300, an epidemic clone of community-acquired meticillin-resistant Staphylococcus aureus. Lancet. 2006;367(9512):731-9.

19. Liu GY. Molecular pathogenesis of Staphylococcus aureus infection. Pediatr Res. 2009;65(5 Pt 2):71R-7R. 
20. Japoni-Nejad A, Rezazadeh M, Kazemian H, Fardmousavi N, van Belkum A, Ghaznavi-Rad E. Molecular characterization of the first community-acquired methicillin-resistant Staphylococcus aureus strains from Central Iran. Int J Infect Dis. 2013;17(11):e949-54.

21. Lamaro-Cardoso J, de Lencastre H, Kipnis A, Pimenta FC, Oliveira LS, Oliveira RM, et al. Molecular epidemiology and risk factors for nasal carriage of staphylococcus aureus and methicillin-resistant S. aureus in infants attending day care centers in Brazil. J Clin Microbiol. 2009;47(12):3991-7.

22. Hashemi SH, Seifrabiei MA, Ahmadi S, Alikhani MY. Frequency of Nasal Carriage of Staphylococcus aureus and Its Antimicrobial Resistance in Hamadan's Medical Students. Sci J Hamadan Univ Med Sci. 2012;19(3):36-40.

23. Ghadiri K, Ebrahimi E, Akramipour R, Rezaei M, Khazaei S, Afsharin MA. Nasal carriage rate of community-and hospital-acquired methicillin-resistant staphylococcus aureus in children, Kermanshah, Iran. Arc Clinl Infect Dis. 2012;6(3):117-20.

24. Choi CS, Yin CS, Bakar AA, Sakewi Z, Naing NN, Jamal F, et al. Nasal carriage of Staphylococcus aureus among healthy adults. J Microbiol Immunol Infect. 2006;39(6):458-64.

25. Yu VL, Goetz A, Wagener M, Smith PB, Rihs JD, Hanchett J, et al. Staphylococcus aureus nasal carriage and infection in patients on hemodialysis. Efficacy of antibiotic prophylaxis. $N$ Engl J Med. 1986;315(2):91-6.

26. Wertheim HFL, Vos MC, Ott A, van Belkum A, Voss A, Kluytmans JAJW, et al. Risk and outcome of nosocomial Staphylococcus aureus bacteraemia in nasal carriers versus non-carriers. Lancet. 2004;364(9435):703-5.

27. VandenBergh MF, Yzerman EP, van Belkum A, Boelens HA, Sijmons M, Verbrugh HA. Follow-up of Staphylococcus aureus nasal carriage after 8 years: redefining the persistent carrier state.J Clin Microbiol.1999;37(10):3133-40.

28. Vasantha Kumari N, Alshrari AS, Rad EG, Moghaddam HG, van Belkum A, Alreshidi MA, et al. Highly dynamic transient colonization by Staphylococcus aureus in healthy Malaysian students. J Med Microbiol. 2009;58(Pt 11):1531-2.

29. Kilic A, Guclu AU, Senses Z, Bedir O, Aydogan H, Basustaoglu AC. Staphylococcal cassette chromosome mec (SCCmec) characterization and panton-valentine leukocidin gene occurrence for methicillin-resistant Staphylococcus aureus in Turkey, from 2003 to 2006. Antonie Van Leeuwenhoek. 2008;94(4):607-14

30. Kirdar S, Arslan U, Tuncer I, Findik D, Bozdogan B. [Investigation of the clonality and Panton-Valentine leukocidin toxin among nosocomial methicillin-resistant Staphylococcus aureus strains]. Mikrobiyol Bul. 2009;43(4):529-33.

31. Tekeli A, Koyuncu E, Dolapci I, Akan OA, Karahan ZC. [Molecular characteristics of methicillin-resistant Staphylococcus aureus strains isolated from blood cultures between 2002-2005 in Ankara University Hospital]. Mikrobiyol Bul. 2009;43(1):1-10.

32. Akoglu H, Zarakolu P, Altun B, Unal S. [Epidemiological and molecular characteristics of hospital-acquired methicillin-resistant Staphylococcus aureus strains isolated in Hacettepe University Adult Hospital in 2004-2005]. Mikrobiyol Bul. 2010;44(3):343-55.

33. Baran CB, Mutlu D, Baysan BO, Gunseren F, Ergani A, Ogunc D, et al. [Investigation of Panton-Valentine leukocidin gene, SCCmec gene cassette types and genotypes of methicillin-resistant Staphylococcus aureus strains isolated from outpatients]. Mikrobiyol Bul. 2010;44(4):533-45.

34. Kuehnert MJ, Kruszon-Moran D, Hill HA, McQuillan G, McAllister SK, Fosheim G, et al. Prevalence of Staphylococcus aureus nasal colonization in the United States, 2001-2002. J Infect Dis. 2006;193(2):172-9.

35. Severin JA, Lestari ES, Kuntaman K, Melles DC, Pastink M, Peeters $\mathrm{JK}$, et al. Unusually high prevalence of panton-valentine leukocidin genes among methicillin-sensitive Staphylococcus aureus strains carried in the Indonesian population. J Clin Microbiol. 2008;46(6):1989-95.

36. Ghasemzadeh-Moghaddam H, Ghaznavi-Rad E, Sekawi Z, YunKhoon L, Aziz MN, Hamat RA, et al. Methicillin-susceptible Staphylococcus aureus from clinical and community sources are genetically diverse. Int J Med Microbiol. 2011;301(4):347-53.

37. Muttaiyah S, Coombs G, Pandey S, Reed P, Ritchie S, Lennon D, et al. Incidence, risk factors, and outcomes of Panton-Valentine leukocidin-positive methicillin-susceptible Staphylococcus aureus infections in Auckland, New Zealand. J Clin Microbiol. 2010;48(10):3470-4.

38. Havaei SA, Vidovic S, Tahmineh N, Mohammad K, Mohsen K, Starnino S, et al. Epidemic methicillin-susceptible Staphylococcus aureus lineages are the main cause of infections at an Iranian university hospital. J Clin Microbiol. 2011;49(11):3990-3.

39. Hoseini Alfatemi SM, Motamedifar M, Hadi N, Sedigh Ebrahim Saraie $\mathrm{H}$. Analysis of Virulence Genes Among Methicillin Resistant Staphylococcus aureus (MRSA) Strains. Jundishapur J Microbiol. 2014;7(6):e10741.

40. Ghaznavi-Rad E, Nor Shamsudin M, Sekawi Z, Khoon LY, Aziz MN, Hamat RA, et al. Predominance and emergence of clones of hospital-acquired methicillin-resistant Staphylococcus aureus in Malaysia.J Clin Microbiol. 2010;48(3):867-72.

41. Barbier F, Lebeaux D, Hernandez D, Delannoy AS, Caro V, Francois P, et al. High prevalence of the arginine catabolic mobile element in carriage isolates of methicillin-resistant Staphylococcus epidermidis. J Antimicrob Chemother. 2011;66(1):29-36.

42. Shore AC, Rossney AS, Brennan OM, Kinnevey PM, Humphreys $\mathrm{H}$, Sullivan DI, et al. Characterization of a novel arginine catabolic mobile element (ACME) and staphylococcal chromosomal cassette mec composite island with significant homology to Staphylococcus epidermidis ACME type II in methicillin-resistant Staphylococcus aureus genotype ST22-MRSA-IV. Antimicrob Agents Chemother. 2011;55(5):1896-905.

43. Ellington MJ, Yearwood L, Ganner M, East C, Kearns AM. Distribution of the ACME-arcA gene among methicillin-resistant Staphylococcus aureus from England and Wales. J Antimicrob Chemother 2008;61(1):73-7.

44. Montgomery CP, Boyle-Vavra S, Daum RS. The arginine catabolic mobile element is not associated with enhanced virulence in experimental invasive disease caused by the community-associated methicillin-resistant Staphylococcus aureus USA300 genetic background. Infect Immun. 2009;77(7):2650-6. 